\title{
Iterative Decision-Directed Joint Frequency Offset and Channel Estimation for KSP-OFDM
}

\author{
Dieter Van Welden, Student Member, IEEE, Heidi Steendam, Senior Member, IEEE, \\ and Marc Moeneclaey, Fellow, IEEE
}

\begin{abstract}
We propose an iterative decision-directed joint frequency offset (FO) and channel estimation algorithm in a known symbol padding (KSP) orthogonal frequency division multiplexing (OFDM) system, where the guard interval is filled with pilot symbols. Besides those time domain pilot symbols, some additional pilot symbols are transmitted on the pilot carriers. The decision-directed algorithm is initialized by pilot-aided FO estimation without channel knowledge. We propose a possible initialization algorithm that operates in the frequency domain (FD). After the initialization phase, the iterative decision-directed estimation algorithm is applied. For the channel estimation step, an existing pilot-aided channel estimation algorithm is extended to a decision-directed algorithm which uses the Fast Fourier Transform outputs at both the pilot and data carrier positions. For the uncoded case, the proposed iterative decision-directed joint FO and channel estimation algorithm reaches the bit error rate performance of a receiver with perfect synchronization and perfect channel knowledge. For a coded system, there is small loss in performance of less than $1 \mathrm{~dB}$ when our proposed algorithm is applied compared to a receiver with perfect knowledge about the FO and the channel.
\end{abstract} tion.

Index Terms-OFDM, channel estimation, frequency estima-

\section{INTRODUCTION}

$\mathbf{O}$ RTHOGONAL Frequency Division Multiplexing (OFDM) [1] has proven to be a valuable technique to achieve a high data rate transmission over multipath fading channels. Various standards are already based on OFDM systems: xDSL [2], digital audio and video broadcasting [3], [4], wireless LAN [5], etc. An OFDM system inserts a guard interval between successively transmitted OFDM blocks to avoid inter block interference. The most popular techniques to fill the guard interval are the cyclic prefix (CP) technique and the zero-padding technique (ZP) (see [6], [7]). In the CP technique, the last samples of each OFDM block are copied and transmitted before the actual OFDM block, whereas the guard interval is left empty in the ZP technique. For these two techniques, there is no control about the content of the guard interval, which makes the guard interval not very useful for channel estimation and sometimes insufficient for synchronization purposes [8]. For example timing synchronization in a CP-OFDM system in

Paper approved by G. E. Corazza, the Editor for Spread Spectrum of the IEEE Communications Society. Manuscript received January 7, 2011; revised August 17, 2011 and March 19, 2012.

The authors are with the Department of Telecommunications and Information Processing, Ghent University, Sint-Pietersnieuwstraat 41, 9000 Gent, Belgium (e-mail: \{dmvwelde, hs, mm $\} @$ telin.ugent.be).

Digital Object Identifier 10.1109/TCOMM.2012.071812.110019 the time domain is usually hard to perform. There exist algorithms that estimate the timing based on the $\mathrm{CP}$, but their performance is degraded in a multipath fading channel (see for example [9]). In this contribution we consider a third guard interval technique called known symbol padding (KSP) [8], which fills the guard interval with pilot symbols. In this technique we have full control about the content of the guard interval, so that it can be useful for synchronization tasks. In [10] for example, a time delay estimation algorithm is proposed for KSP-OFDM that outperforms existing time delay estimation algorithms for CP-OFDM. There exist channel estimation algorithms for KSP-OFDM which can take advantage of the guard interval, but they have a slightly higher computational complexity than channel estimation algorithms for CP-OFDM (see for example [11]). For the bit error rate (BER), CP-OFDM and ZP-OFDM achieve both the same performance [6], [7]. The performance of a KSP-OFDM system is slightly worse: there is a degradation of $10 \log (1+\gamma) \mathrm{dB}$ in $E_{b} / N_{0}$ [7], where $\gamma$ is the ratio of the duration of the guard interval and the duration of the useful part of the OFDM block.

In the literature, a special version of KSP-OFDM is called time domain synchronous (TDS) OFDM and has been applied in the Chinese digital television standard [12]. In a TDSOFDM system the guard interval has a special structure: it consists of a pre-amble, a pseudo noise sequence and a post-amble. There are no extra pilot symbols available in the frequency domain. In this paper we consider a more general KSP-OFDM system with pilot symbols in the guard interval and some additional pilot symbols that are transmitted on the pilot carriers. The main advantage of the extra pilot carriers is that channel estimation can be performed in the frequency domain, where, after synchronization, the contributions of the pilot symbols and the data symbols can be perfectly separated. As a result a channel estimate that does not show an error floor in terms of the MSE can be obtained (see for example [13]).

One of the problems in an OFDM system is its sensitivity to a frequency mismatch between the oscillators in the transmitter and the receiver. The bit error rate (BER) performance can be severely degraded when the frequency offset (FO) (even of the order of a fraction of the carrier spacing) is not compensated [14], because the FO causes inter-carrier interference and attenuates the useful signal. In the literature several FO estimation algorithms for TDS-OFDM can be found. In [15], it is shown that the content of the guard interval in a TDS-OFDM system can be viewed as a CP-OFDM 
training symbol. The algorithm from [9] is then applied. The authors from [16] introduce the method of composite PNcorrelation, which computes the point-wise conjugate product of two phase-shifted PN-correlations. The obtained product is then used to estimate the FO. In [17], the guard interval of one OFDM block is correlated with the guard interval of the next OFDM block. When the channel varies slowly during two consecutive OFDM blocks, there is only a difference in phase between the two received guard intervals. This phase difference depends linearly on the FO, so it can be exploited to estimate the FO. These algorithms for a TDS-OFDM system only make use of the time domain (TD) pilot symbols while there are also frequency domain (FD) pilot symbols available in a general KSP-OFDM system.

We present in this contribution an iterative decision-directed joint FO and channel estimation algorithm which iterates between FO estimation, channel estimation and data detection. For the channel estimation step, we extend the pilot-aided algorithm proposed in [13]. The iterative decision-directed algorithm is initialized by a pilot-aided FO estimation algorithm without any knowledge about the channel.

Besides the already mentioned TDS-OFDM algorithms which can be applied to initialize our iterative decisiondirected algorithm, we propose a new initialization algorithm that operates in the FD. The algorithm exploits the presence of the pilot symbols on the pilot carriers (FD pilots) and in the guard interval (TD pilots), both forming part of the received signal that corresponds to two or more consecutively transmitted OFDM blocks.

The initialization algorithm shows an error floor for the mean squared error (MSE). After the initialization phase the decision-directed algorithm is applied. It turns out that when no error correcting code is applied, the proposed decisiondirected algorithm reaches the BER performance of a receiver with perfect synchronization and channel state information (CSI) after one iteration, regardless of which initial estimator is used to obtain a first estimate of the FO. For a coded system, there is a gap of less than $1 \mathrm{~dB}$ between the performance of a receiver with perfect synchronization and perfect channel knowledge and a receiver which applies our proposed algorithm.

\section{System Model}

Consider a KSP-OFDM system with $N$ carriers and a guard interval consisting of $\nu$ samples. The transmitted symbols on the carriers during the $i$-th OFDM block are denoted as $\mathbf{a}_{i}=\left(a_{i}(0), \ldots, a_{i}(N-1)\right)^{T}$. The vector $\mathbf{a}_{i}$ contains $M-\nu$ FD pilot symbols denoted as $\mathbf{b}_{c}=$ $\left(b_{c}(0), \ldots, b_{c}(M-\nu-1)\right)^{T}$ and $N-M+\nu$ data symbols denoted as $\mathbf{a}_{d}^{(i)}=\left(a_{d}^{(i)}(0), \ldots, a_{d}^{(i)}(N-M+\nu-1)\right)^{T}$. The set of carrier indices is divided in two subsets $S_{p}=$ $\left\{\alpha_{0}, \ldots, \alpha_{M-\nu-1}\right\}$, which is the set of pilot carriers, and $S_{d}=\left\{\beta_{0}, \ldots, \beta_{N+\nu-M-1}\right\}$, which contains the data carriers. The guard interval is filled with $\nu$ (TD) pilot symbols denoted as $b_{g}=\left(b_{g}(0), \ldots, b_{g}(\nu-1)\right)^{T}$, so the total number of transmitted pilot symbols per OFDM block is equal to $M$. After the inverse Fast Fourier Transform (IFFT) and the insertion of the guard interval, we obtain the $N+\nu$ transmitted a) transmitter
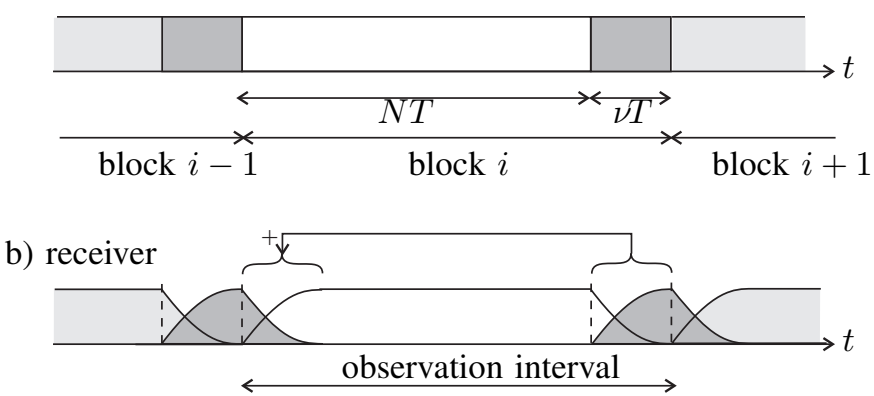

Fig. 1. Time-domain signal of KSP-OFDM a) transmitted signal b) received signal and observation interval.

time-domain samples during the $i$-th block:

$$
\mathbf{s}_{i}=\sqrt{\frac{N}{N+\nu}}\left(\begin{array}{c}
\mathbf{F}^{H} \mathbf{a}_{i} \\
\mathbf{b}_{g}
\end{array}\right),
$$

where $\mathbf{F}$ is the $N \times N$ FFT matrix with $\mathbf{F}_{k, l}=$ $(1 / \sqrt{N}) e^{-j 2 \pi \frac{k l}{N}}$. The data symbols are assumed to be independent and identically distributed (i.i.d.) and $E\left[\left|a_{d}^{(i)}\right|\right]^{2}=$ $E_{s}$. Further we assume that $E\left[\left|b_{c}(n)\right|^{2}\right]=E\left[\left|b_{g}(k)\right|^{2}\right]=$ $E_{s}$. The normalization factor $\sqrt{N /(N+\nu)}$ in (1) guarantees that $E\left[\left|\mathbf{s}_{i}\right|^{2}\right]=N E_{s}$. We can rewrite $\mathbf{s}_{i}$ as the sum of a vector $\mathbf{s}_{p}$, which collects the contributions from both the pilot symbols in time and frequency domain, and a vector $\mathbf{s}_{d}^{(i)}$, which consists of the contributions from the unknown data symbols:

$$
\mathbf{s}_{i}=\mathbf{s}_{p}+\mathbf{s}_{d}^{(i)} .
$$

The OFDM blocks are transmitted over a frequency selective channel with impulse response $\mathbf{h}=(h(0), \ldots, h(L-1))^{T}$ where $L$ denotes the number of channel taps. To avoid inter block interference, we select $\nu \geq L-1$. The $N+\nu$ received time-domain samples corresponding to the observation interval shown in Fig. 1.b are given by

$$
\mathbf{r}_{i}=e^{j 2 \pi \frac{i(N+\nu)}{N} \epsilon} \mathbf{E}(\epsilon) \tilde{\mathbf{H}} \mathbf{s}_{i}+\mathbf{w}_{i} .
$$

The unknown parameter $\epsilon$ denotes the FO between the transmitter and receiver oscillators expressed as a fraction of the intercarrier spacing. We assume that the timing offset is already known and that a rough estimation of the FO already has been performed so that $|\epsilon|<0.5 N /(N+\nu)$. The $(N+\nu) \times(N+\nu)$ channel matrix $\tilde{\mathbf{H}}$ is given by

$$
(\tilde{\mathbf{H}})_{k, k^{\prime}}=h\left(\left|k-k^{\prime}\right|_{N+\nu}\right)
$$

where $|x|_{K}$ is the notation of the modulo- $K$ operation which gives the remainder of the division of $x$ by $K$. The diagonal matrix $\mathbf{E}$ is defined as: $(\mathbf{E}(\epsilon))_{k, k}=e^{j 2 \pi \frac{k}{N} \epsilon}$. The channel also adds additive white Gaussian noise $\mathbf{w}_{i}$. The real and imaginary parts of the components of $\mathbf{w}_{i}$ are i.i.d. with zero mean, and variance $N_{0} / 2$.

To detect the transmitted data symbols of OFDM block $i$ a sequence of operations must be carried out. First, the FO 
is compensated in the time domain using the FO estimate. Then the contributions from the guard interval samples are subtracted from the received signal samples using the channel estimate. The resulting received signal is similar to a received signal from a ZP-OFDM system. The last $\nu$ samples of the considered observation interval in Fig. 1 are added to the first $\nu$ samples, to restore the orthogonality between the carriers (see [6]). Note that after the removal of the guard interval samples, the resulting first $N$ samples are applied to an FFT to transform the frequency selective channel in $N$ parallel flat fading channels. Next, per carrier single-tap equalization is performed using the channel estimate. Finally, the equalizer outputs are applied to the detector/decoder. The iterative decision-directed algorithm to jointly estimate $\epsilon$ and $\mathbf{h}$ is introduced in the next section.

\section{FREQUENCY OFFSET ESTIMATION}

Our proposed algorithm starts from the received signal samples of $K(\geq 2)$ consecutively transmitted OFDM blocks. We denote them as $\mathbf{r}_{0}, \ldots, \mathbf{r}_{K-1}$, which are given by (3):

$$
\begin{aligned}
\mathbf{r}_{0} & =\mathbf{E}(\epsilon) \tilde{\mathbf{H}}\left(\mathbf{s}_{p}+\mathbf{s}_{d}^{(0)}\right)+\mathbf{w}_{0} \\
& \vdots \\
\mathbf{r}_{K-1} & =e^{j 2 \pi \frac{(K-1)(N+\nu)}{N} \epsilon} \mathbf{E}(\epsilon) \tilde{\mathbf{H}}\left(\mathbf{s}_{p}+\mathbf{s}_{d}^{(K-1)}\right)+\mathbf{w}_{K-1}
\end{aligned}
$$

We see that the pilot symbol contribution in $\mathbf{r}_{i}$ (i.e. the contribution from $\mathbf{s}_{p}$ ), with $i=1, \ldots, K-1$, is equal to the pilot symbol contribution in $\mathbf{r}_{0}$ multiplied by a factor $e^{j 2 \pi \frac{i(N+\nu)}{N} \epsilon}$. We will exploit this fact to estimate the FO $\epsilon$.

First we transform the received vectors $\mathbf{r}_{i}, i=0, \ldots, K-$ 1 to the frequency domain. To achieve this, the last $\nu$ samples of each vector are added to the first $\nu$ samples. The first $N$ samples of the resulting vectors are then applied to an FFT. The output of the FFT of OFDM block $i$ at carrier $n$ is given by

$$
\begin{aligned}
Y_{i}(n)= & e^{j 2 \pi \frac{i(N+\nu)}{N} \epsilon}\left(H(\epsilon, n) \sum_{m=0}^{M-\nu-1} b_{c}(m) I_{\alpha_{m}-n}(\epsilon)\right. \\
& +H(\epsilon, n) \sum_{m=0}^{N+\nu-M-1} a_{d}^{(i)}(m) I_{\beta_{m}-n}(\epsilon) \\
& \left.+\sum_{l=0}^{L-1} B_{g}(\epsilon, n, l) h(l)\right)+W_{i}(n)
\end{aligned}
$$

where $I_{m}(\epsilon), H(\epsilon, n)$ and $B_{g}(\epsilon, n, l)$ are given by

$$
\begin{aligned}
I_{m}(\epsilon)= & \frac{1}{\sqrt{N(N+\nu)}} \frac{1-e^{j 2 \pi(m+\epsilon)}}{1-e^{j 2 \pi \frac{(m+\epsilon)}{N}}} \\
H(\epsilon, n)= & \sum_{l=0}^{L-1} h(l) e^{-j 2 \pi \frac{(n-\epsilon) l}{N}} \\
B_{g}(\epsilon, n, l)= & \frac{1}{\sqrt{N+\nu}}\left(\sum_{k=0}^{l-1} b_{g}(\nu+k-l) e^{j 2 \pi \frac{(\epsilon-n) k}{N}}\right. \\
& \left.+\sum_{k=l}^{\nu-1} b_{g}(k-l) e^{j 2 \pi \frac{(\epsilon-n)(N+k)}{N}}\right),
\end{aligned}
$$

and $\alpha_{m}$ and $\beta_{m}$ are carrier indices belonging to the subsets of carrier indices of pilot carriers and data carriers, respectively. The samples $W_{i}(n)$, with $n=0, \ldots, N-1$, are Gaussian noise samples with zero mean and an autocorrelation matrix $\mathbf{R}$ defined as

$$
(\mathbf{R})_{n, n^{\prime}}=N_{0}\left(\delta_{n, n^{\prime}}+\frac{1}{N} \sum_{k=0}^{\nu-1} e^{-j 2 \pi \frac{\left(n-n^{\prime}\right) l}{N}}\right) .
$$

The next subsection describes the proposed decisiondirected FO estimation algorithm.

\section{A. Decision-Directed FO Estimation}

Initially, an estimate of the FO $\hat{\epsilon}_{0}$ is obtained by applying some FO estimation algorithm (we suggest two possible algorithms in Subsections III-B and III-C). This estimate is used to compensate the FO in the time domain. In a second step, the resulting signal is transformed to the frequency domain to estimate the channel impulse response $\mathbf{h}$ using the algorithm from [13]. The channel estimate is then used to detect the transmitted data symbols. We can now start an iterative algorithm which iterates between the following processes: i) obtaining an estimate of the remaining FO using the latest obtained estimate of $\mathbf{h}$ and the detected data symbols, ii) re-estimating $\mathbf{h}$ after compensation of the remaining FO and using the detected data symbols, and iii) refining the detection of the data symbols using the most recent estimate of $\mathbf{h}$. This procedure is repeated until convergence is reached.

In the $p$-th iteration, the remaining FO is denoted as $\epsilon_{p}$ and defined as

$$
\epsilon_{p}=\epsilon-\sum_{k=0}^{p-1} \hat{\epsilon}_{k} .
$$

The FFT output of OFDM block $i$ at carrier $n$, denoted by $Y_{i}^{(p)}(n)$, is given by (5) where we replace $\epsilon$ by $\epsilon_{p}$. When $\epsilon_{p}$ is very small, the inter-carrier interference can be neglected: $I_{m}\left(\epsilon_{p}\right) \approx 0$, for $m \neq 0$, which yields for the FFT outputs at the pilot carrier positions:

$$
\begin{aligned}
Y_{i}^{(p)}\left(\alpha_{m}\right) & =e^{j 2 \pi \frac{i(N+\nu)}{N} \epsilon_{p}}\left(b_{c}(m) I_{0}\left(\epsilon_{p}\right) H\left(\epsilon_{p}, \alpha_{m}\right)\right. \\
& \left.+\sum_{l=0}^{L-1} B_{g}\left(\epsilon_{p}, \alpha_{m}, l\right) h(l)\right)+W_{i}^{(p)}\left(\alpha_{m}\right),
\end{aligned}
$$

where $\alpha_{m} \in S_{p}$. The signal component of $Y_{i}^{(p)}\left(\alpha_{m}\right)$ is the product of a factor which depends on the block index $i$ and a factor which is independent of the block index. We exploit this fact to estimate the FO.

The FFT outputs at the data carrier positions are given by

$$
\begin{aligned}
Y_{i}^{(p)}\left(\beta_{m}\right) & =e^{j 2 \pi \frac{i(N+\nu)}{N} \epsilon_{p}}\left(a_{d}^{(i)}(m) I_{0}\left(\epsilon_{p}\right) H\left(\epsilon_{p}, \beta_{m}\right)\right. \\
& \left.+\sum_{l=0}^{L-1} B_{g}\left(\epsilon_{p}, \beta_{m}, l\right) h(l)\right)+W_{i}^{(p)}\left(\beta_{m}\right)
\end{aligned}
$$

where $\beta_{m} \in S_{d}$. The signal component of $Y_{i}^{(p)}\left(\beta_{m}\right)$ can not be expressed as the product of a factor which depends on the block index and a factor which is independent of it, so we have 
to modify the FFT outputs at the data carrier positions first. There are two possible options: i) Subtracting the contribution from the unknown data symbols (using previous data symbol decisions), or ii) subtracting the contribution from the guard interval pilot symbols and multiplying the results with the complex conjugates of the latest obtained hard decisions of the unknown data symbols. One can prove that the power ratio of the data symbols and the guard interval pilot symbols contribution in $Y_{i}^{(p)}\left(\beta_{m}\right)$ is approximately equal to $N / \nu$. Since $N$ is usually much larger than $\nu$, the second option will lead to a smaller estimation error as the variance of the guard interval pilots is smaller. Therefore, we choose to follow the second option.

First, we have to subtract the contribution from the guard interval pilot samples ${ }^{1}$ :

$$
Z_{i}^{(p)}\left(\beta_{m}\right)=Y_{i}^{(p)}\left(\beta_{m}\right)-\sum_{l=0}^{L-1} B_{g}\left(0, \beta_{m}, l\right) \hat{h}^{(p-1)}(l),
$$

where $\beta_{m} \in S_{d}$ and $\hat{h}^{(p-1)}(l), l=0, \ldots, L-1$, are the estimated samples of the channel impulse response obtained in the $(p-1)$-th iteration. We assume that the estimated channel impulse response is equal to the true channel impulse response so that the contribution of the guard interval can be perfectly removed:

$$
\begin{aligned}
Z_{i}^{(p)}\left(\beta_{m}\right)=e^{j 2 \pi \frac{i(N+\nu)}{N} \epsilon_{p}} a_{d}^{(i)}(m) I_{0}\left(\epsilon_{p}\right) & H\left(\epsilon_{p}, \beta_{m}\right) \\
& +W_{i}^{(p)}\left(\beta_{m}\right) .
\end{aligned}
$$

Multiplying $Z_{i}^{(p)}\left(\beta_{m}\right)$ by the complex conjugate of the latest obtained hard decision of $a_{d}^{(i)}(m)$ results in

$$
\begin{aligned}
Z_{i}^{\prime(p)}\left(\beta_{m}\right)=e^{j 2 \pi \frac{i(N+\nu)}{N} \epsilon_{p}} & E_{s} I_{0}\left(\epsilon_{p}\right) H\left(\epsilon_{p}, \beta_{m}\right) \\
& +\left(a_{d}^{(i)}(m)\right)^{*} W_{i}^{(p)}\left(\beta_{m}\right),
\end{aligned}
$$

where we assume that the decisions about the data symbols from the previous iteration are correct, and $z^{*}$ denotes the complex conjugate of $z$.

Both (6) and (8) correspond to the following model:

$$
U_{i}(n)=e^{j 2 \pi \frac{i(N+\nu)}{N} \epsilon_{p}} \Gamma(n)+V_{i}(n),
$$

where $\Gamma(n)$ can be seen as an unknown coefficient and $V_{i}(n)$ is the additive noise. Rewriting everything in vector format yields

$$
\mathbf{U}_{i}=e^{j 2 \pi \frac{i(N+\nu)}{N} \epsilon_{p}} \boldsymbol{\Gamma}+\mathbf{V}_{i}
$$

The next step is to obtain the least squares estimates of $\epsilon_{p}$ and $\boldsymbol{\Gamma}$, which are given by

$$
\left(\hat{\epsilon}_{p}, \hat{\boldsymbol{\Gamma}}\right)=\arg \min _{\epsilon_{p}, \boldsymbol{\Gamma}} \sum_{i=0}^{K-1}\left|\mathbf{U}_{i}-e^{j 2 \pi \frac{i(N+\nu)}{N} \epsilon_{p}} \boldsymbol{\Gamma}\right|^{2} .
$$

The estimate $\hat{\boldsymbol{\Gamma}}$ can be expressed as a function of $\epsilon_{p}$ and is given by

$$
\hat{\boldsymbol{\Gamma}}\left(\epsilon_{p}\right)=\frac{1}{K} \sum_{i=0}^{K-1} e^{-j 2 \pi \frac{i(N+\nu)}{N} \epsilon_{p}} \mathbf{U}_{i} .
$$

\footnotetext{
${ }^{1}$ Again we assume that $\epsilon_{p}$ is small: $e^{j 2 \pi \frac{i(N+\nu)}{N} \epsilon_{p}} B_{g}\left(\epsilon_{p}, \beta_{m}, l\right) \approx$ $B_{g}\left(0, \beta_{m}, l\right)$.
}

Substituting $\Gamma$ by its estimate (10) in (9) yields

$$
\hat{\epsilon}_{p}=\arg \max _{\epsilon_{p}}\left|\sum_{i=0}^{K-1} e^{-j 2 \pi \frac{i(N+\nu)}{N} \epsilon_{p}} \mathbf{U}_{i}\right|^{2}
$$

where we have omitted irrelevant terms and a constant factor. Rearranging the terms of the squared magnitude results $i^{2}$

$$
\hat{\epsilon}_{p}=\arg \max _{\epsilon_{p}} \sum_{m=1}^{K-1} 2 \Re\left\{R(m) e^{-j 2 \pi \frac{N+\nu}{N} m \epsilon_{p}}\right\}
$$

where the correlation $R(m)$ is defined as

$$
R(m)=\sum_{i=0}^{K-1-m} \mathbf{U}_{i}^{H} \mathbf{U}_{i+m} .
$$

Now we differentiate the function from (11) with respect to $\epsilon_{p}$ and equate the derivative to zero:

$$
\sum_{m=1}^{K-1} \Im\left\{m R(m) e^{-j 2 \pi \frac{N+\nu}{N} m \epsilon_{p}}\right\}=0 .
$$

This can be rewritten as

$$
\sum_{m=1}^{K-1} m|R(m)| \sin \left[\angle(R(m))-2 \pi \frac{N+\nu}{N} m \epsilon_{p}\right]=0,
$$

where $\angle(x)$ returns the argument of the complex number $x$ in the interval $[-\pi, \pi[$. We assume that the noise contribution in $R(m)$ is small so that $\angle(R(m)) \approx 2 \pi \frac{N+\nu}{N} m \epsilon_{p}$. As the function $\sin (x)$ can be approximated by $x$ for small values of $x$, expression (13) can be approximated by

$$
\sum_{m=1}^{K-1} m|R(m)|\left(\angle(R(m))-2 \pi \frac{N+\nu}{N} m \epsilon_{p}\right)=0 .
$$

Solving this equation with respect to $\epsilon_{p}$ yields the estimate of $\epsilon_{p}$. To expand the range of possible values of $\epsilon_{p}$ that can be estimated and / or to reduce the numerical complexity of the algorithm, the interval of the summation index $m$ can be limited to $\left[P_{1}, P_{2}\right]$, where $1 \leq P_{1} \leq P_{2} \leq K-1$. The estimate of $\epsilon_{p}$ is then given by

$$
\hat{\epsilon}_{p}=\frac{1}{2 \pi} \frac{N}{N+\nu} \frac{\sum_{m=P_{1}}^{P_{2}} m|R(m)| \angle(R(m))}{\sum_{m=P_{1}}^{P_{2}} m^{2}|R(m)|} .
$$

A special case of the algorithm is obtained when we choose $P_{1}=P_{2}=P$, where $1 \leq P \leq K-1$. This yields for the estimate of $\epsilon_{p}$ :

$$
\hat{\epsilon}_{p}=\frac{1}{2 \pi} \frac{N}{N+\nu} \frac{\angle(R(P))}{P} .
$$

In the next step, the remaining FO is compensated using the estimate $\hat{\epsilon}_{p}$ and a new estimate of the channel is obtained per block $i, i=0, \ldots, K-1$, by applying a channel estimation algorithm which is an extension of the algorithm from [13]: We assume that the obtained hard decisions of the unknown data symbols are correct and we apply the algorithm from [13], but instead of only selecting the pilot carriers, we use both the pilot and the data carriers to obtain an estimate of $\mathbf{h}$.

\footnotetext{
${ }^{2}$ We have omitted the term for $m=0$, because it is independent of $\epsilon_{p}$
} 


\section{B. TD Initialization}

As already mentioned in the introduction, there exist some FO estimation algorithms for a TDS-OFDM system which could be applied for a KSP-OFDM. They can provide the initial estimate of the FO. Especially the algorithm from [17] comes in mind because it can be applied directly without any changes.

The algorithm starts from the received signal vectors corresponding to the guard interval $\mathbf{r}_{1, i}=\left(r_{i}(N), \ldots, r_{i}(N+\nu-1)\right)^{T}, i=0, \ldots, K-1$, which are defined as

$$
\mathbf{r}_{1, i}=e^{j 2 \pi \frac{i(N+\nu)}{N} \epsilon} \mathbf{E}_{1}(\epsilon) \tilde{\mathbf{H}}_{g}\left(\mathbf{s}_{p}+\mathbf{s}_{d}^{(i)}\right)+\mathbf{w}_{1, i}
$$

where $\tilde{\mathbf{H}}_{g}$ consists of the last $\nu$ rows of $\tilde{\mathbf{H}}, \mathbf{E}_{1}(\epsilon)$ is a diagonal matrix given by $\left(\mathbf{E}_{1}(\epsilon)\right)_{k, k}=e^{j 2 \pi \frac{N+k}{N} \epsilon}$ and $\mathbf{w}_{1, i}$ collects the noise samples. We compute the product of the Hermitian transpose of $\mathbf{r}_{1, i}$ with $\mathbf{r}_{1, i+1}$ for $i=0, \ldots, K-2$. The summation of those $K-1$ products yields a quantity that can be used to estimate $\epsilon$ :

$$
\sum_{i=0}^{K-2} \mathbf{r}_{1, i}^{H} \mathbf{r}_{1, i+1}=(K-1) e^{j 2 \pi \frac{N+\nu}{N} \epsilon}\left|\tilde{\mathbf{H}} \mathbf{s}_{p}\right|^{2}+\sum_{i=0}^{K-2} n_{i}
$$

where $n_{i}$ collects the contributions from $\mathbf{r}_{1, i}^{H} \mathbf{r}_{1, i+1}$ which depend on the noise and the unknown data symbols. The estimate of $\epsilon$ is then given by

$$
\hat{\epsilon}_{0}=\frac{1}{2 \pi} \frac{N}{N+\nu} \angle\left\{\sum_{i=0}^{K-2} \mathbf{r}_{1, i}^{H} \mathbf{r}_{1, i+1}\right\} .
$$

Besides the TD pilot symbols in the guard interval there are also pilot symbols available on the pilot carriers which can be used to estimate the FO. Therefore, a straightforward extension of the algorithm from [17] would be to consider the total received symbol vectors $\mathbf{r}_{i}$ instead of only the parts corresponding to the guard intervals $\mathbf{r}_{1, i}$. However, using the received signals that correspond to the guard intervals results in a much higher signal-to-noise ratio than using the total vectors $\mathbf{r}_{i}$. So applying the algorithm from [17] to the received symbol vectors $\mathbf{r}_{i}$ results in a less accurate estimate and a degradation of the performance. To exploit also the pilot carriers besides the pilot symbols in the guard interval, a different approach has to be considered.

\section{FD Initialization}

We collect the $M-\nu$ FFT outputs from the $K$ OFDM blocks corresponding to the set $S_{p}$ of pilot carriers in the vectors $\mathbf{y}_{i}, i=0, \ldots, K-1$ :

$$
\begin{aligned}
\mathbf{y}_{i} & =\left[Y_{i}\left(\alpha_{0}\right), \ldots, Y_{i}\left(\alpha_{M-\nu-1}\right)\right]^{T} \\
& =e^{j 2 \pi \frac{i(N+\nu)}{N} \epsilon}\left(\tilde{\mathbf{b}}+\tilde{\mathbf{a}}_{i}\right)+\mathbf{W}_{i}
\end{aligned}
$$

where $\tilde{\mathbf{b}}$ is the vector which contains the contributions from both time and frequency domain pilots. The vector $\tilde{\mathbf{a}}_{i}$ collects the contributions from the unknown data symbols from the $i$-th OFDM block and $\mathbf{W}_{i}$ contains the noise samples at the pilot carrier positions of the $i$-th block. The summation over $i$ of the multiplications of the Hermitian transpose of $\mathbf{y}_{i}$ with
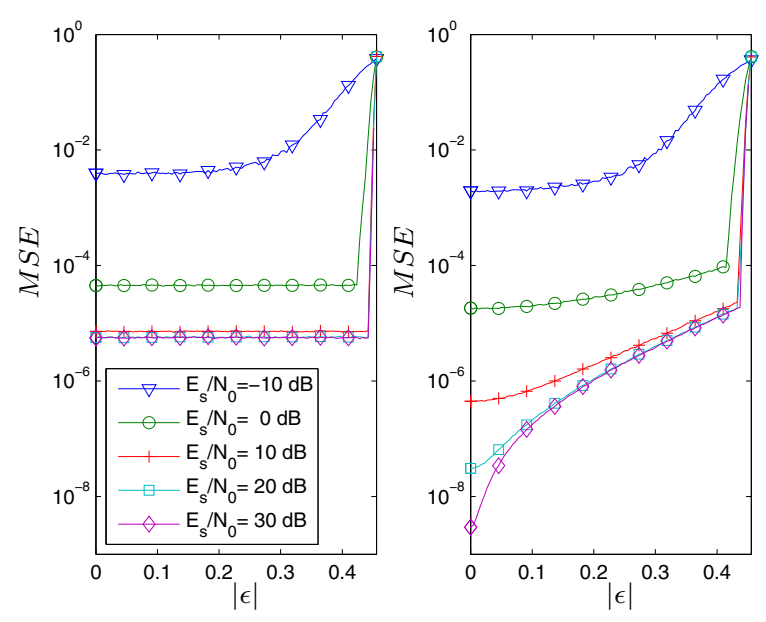

Fig. 2. MSE versus $|\epsilon|$ for TD (left) and FD (right) initialization, $\nu=100$, $N=1024, M=200, K=10$.

$\mathbf{y}_{i+1}$ for $i=0, \ldots, K-2$ results in a function which is used to estimate $\epsilon$ :

$$
\sum_{i=0}^{K-2} \mathbf{y}_{i}^{H} \mathbf{y}_{i+1}=(K-1) e^{j 2 \pi \frac{(N+\nu)}{N} \epsilon}|\tilde{\mathbf{b}}|^{2}+\sum_{i=0}^{K-2} \tilde{n}_{i}
$$

where $\tilde{n}_{i}$ collects the contributions from the unknown data symbols and the noise samples from $\mathbf{y}_{i}^{H} \mathbf{y}_{i+1}$. The estimate of $\epsilon$ is then given by

$$
\hat{\epsilon}_{0}=\frac{1}{2 \pi} \frac{N}{N+\nu} \angle\left\{\sum_{i=0}^{K-2} \mathbf{y}_{i}^{H} \mathbf{y}_{i+1}\right\}
$$

\section{Simulation Results And Discussion}

In this section, the performance of the proposed frequency estimation algorithms is evaluated. We consider a frequency selective Rayleigh fading channel consisting of $L=50$ channel taps with equal variance. The channel impulse response is normalized $\sum_{l=0}^{L-1} E\left[|h(l)|^{2}\right]=1$. For the pilots transmitted on the carriers, the comb-type pilot arrangement from [18] is used. The pilot symbols are randomly selected QPSK symbols. The pilot symbols are equally spaced over the pilot carriers: $S_{p}=\{m \lambda \mid m=0, \ldots, M-\nu-1\}$, where $\lambda=$ floor $(N /(M-\nu))$. We consider an OFDM system with $N=1024$ carriers and a guard interval of length $\nu=100$. Besides the 100 pilot symbols in the guard interval, an additional 100 carriers are selected as pilot carriers which means that a total number of $M=200$ pilot symbols are transmitted. The data symbols are QPSK symbols. For the FO estimation we consider $K=10$ received OFDM blocks.

First we evaluate the performance of the estimators in terms of the MSE, which is defined as:

$$
\operatorname{MSE}(p)=E\left[\left|\epsilon-\sum_{k=0}^{p} \hat{\epsilon}_{k}\right|^{2}\right]
$$

where $p$ is the iteration number.

Fig. 2 shows the MSE of the TD algorithm from [17] and our proposed FD initialization algorithm versus $|\epsilon|$ for different values of $E_{s} / N_{0}$. We see that both estimators have 


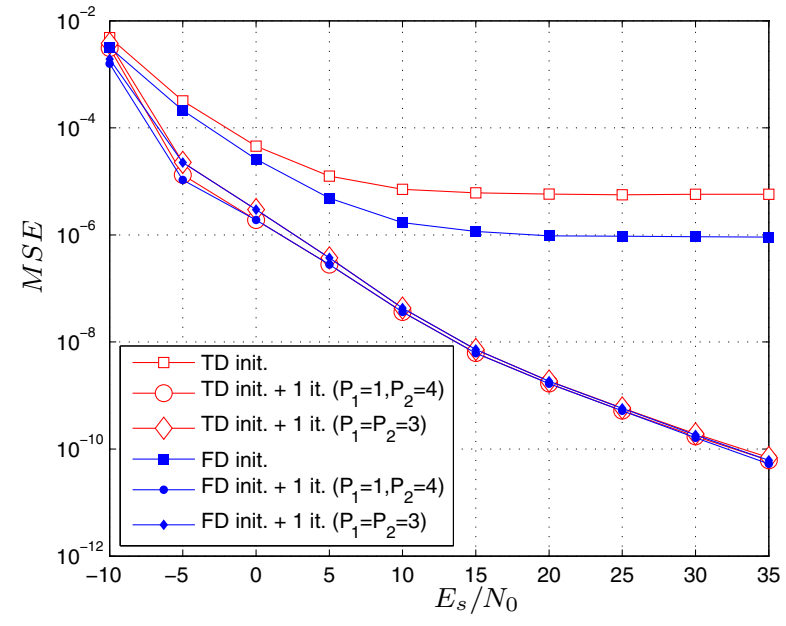

Fig. 3. MSE versus $E_{s} / N_{0}, \nu=100, N=1024, M=200, K=10$.

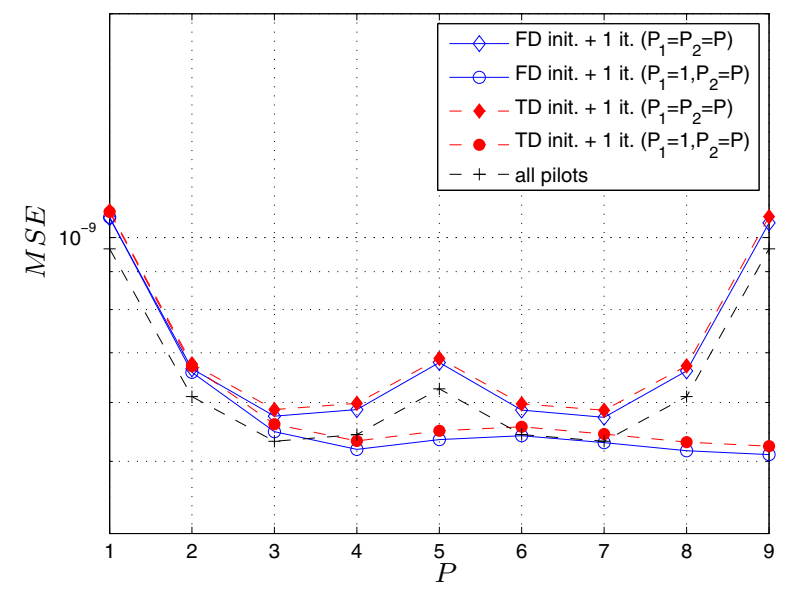

Fig. 4. MSE versus $P$, for $E_{s} / N_{0}=25 \mathrm{~dB}, \nu=100, N=1024$, $M=200, K=10$.

a bad performance for $|\epsilon|>0.3$. This is due to the discontinuity of the $\angle\{$.$\} function in (18) and (19). Therefore$ for the following simulation results we will assume that $\epsilon$ is randomly selected from a uniform distribution over the interval $[-0.3,0.3]$. For the TD initialization we see that the MSE is only weakly dependent on $\epsilon$ and for high signal-to-noise ratios (SNR) independent of the SNR. The performance of the FD initial estimator on the contrary, strongly depends on the value of $\epsilon$, especially for high SNR. For high values of $\epsilon$, the MSE becomes independent of the SNR. As a result, both estimators will show an error floor for high values of the SNR when we plot the MSE versus the $E_{s} / N_{0}$, as can be seen from Fig. 3. This error floor is caused by the unknown data symbols which interfere with the pilot symbols. We see that the FD initialization yields the lowest error floor. This behavior is expected because for very small values of $\epsilon$, the ICI will become very small. In that case the interference of the unknown data symbols will be much smaller in the FD (and eventually equal to zero when $\epsilon=0$ ), while the interference in the TD is independent of the presence of a FO.

To evaluate the performance of the decision-directed FO

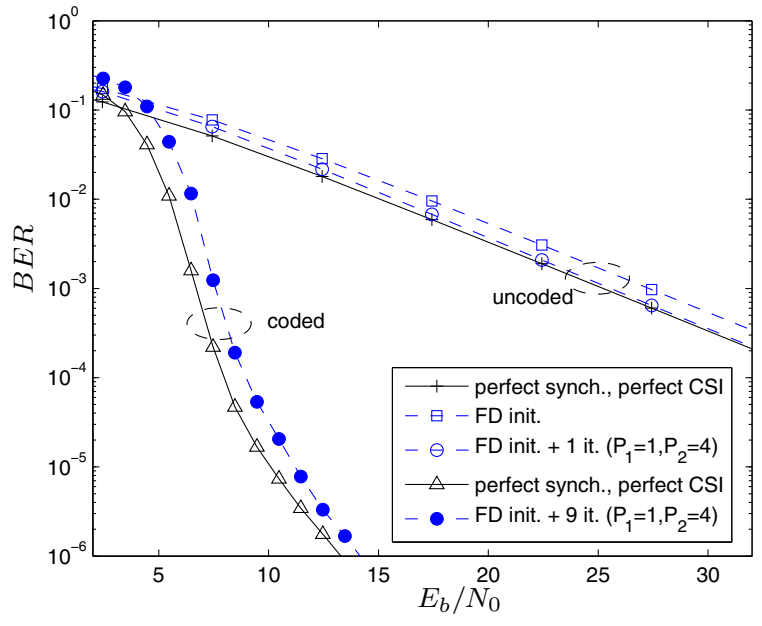

Fig. 5. BER results, $\nu=100, N=1024, M=200, K=10$.

estimation algorithm, we have first done some simulations to determine the optimal values for the parameters $P_{1}$ and $P_{2}$. We have considered two situations:

- in the first case we select $P_{1}$ equal to 1 and let $P_{2}$ be equal to $P$,

- in the second case we select both $P_{1}$ and $P_{2}$ equal to $P$. The parameter $P$ varies from 1 to $K-1$. Fig. 4 shows the MSE as a function of $P$ for $E_{s} / N_{0}=25 \mathrm{~dB}$. For the first case, the lowest MSE is reached when $P_{2}$ is chosen equal to $K-1=9$, which is the maximum value for $P_{2}$. The curve is almost independent of $P$ when $P \in[4,9]$, so choosing $P_{2}=4$ instead of the optimal value 9 , results in a near optimum performance in terms of the MSE while the numerical complexity is reduced.

For the second case, the performance of the estimator is optimal when both $P_{1}$ and $P_{2}$ are chosen equal to 3 or 7 . To explain this, we look at the performance of the all pilots case, also shown in the figure. It can be observed that the performance in the second case is (essentially) proportional to that of the all pilots case. In the appendix the MSE performance of the all pilots case is derived. The minimum value of the MSE is indeed reached for $P_{1}$ and $P_{2}$ both equal to the values of $P$ closest to $10 / 3$ and $20 / 3$. The second case gives rise to a small loss in performance compared to the first case. These results are independent of which initialization algorithm was used first.

The MSE of the decision-directed FO estimation algorithm as a function of $E_{s} / N_{0}$ is also shown in Fig. 3. The results are shown for $P_{1}=1$ and $P_{2}=4$; and $P_{1}=P_{2}=3$. Compared to the results of the initialization algorithms, a large improvement is reached after only one iteration: independent of which initialization algorithm has been applied, the error floor is completely removed for the considered range of $E_{s} / N_{0}$ values.

Both considered choices for $P_{1}$ and $P_{2}$ result in a similar performance. Only for low to moderate $E_{s} / N_{0}$, there is a small difference in performance.

Fig. 5 shows the BER results as a function of $E_{b} / N_{0}$, where $E_{b}$ is the transmitted energy per information bit. We 
have only considered our proposed FD initialization method to obtain these results, because we have seen from Fig. 3 that the performance of our proposed decision-directed algorithm is independent of the initialization algorithm. For the uncoded case, the BER of a receiver which only applies the FD initialization algorithm for the estimation of the FO, is close to the case when the FO is perfectly known (but with estimated channel).

After one iteration, the decision-directed algorithm results in a performance which is close to the BER of a receiver with perfect knowledge about the channel and the FO. Additional iterations are not necessary for the considered range of $E_{s} / N_{0}$ values, as the potential improvements are negligible.

During the derivation of the iterative decision-directed algorithm, we have made some assumptions concerning the correctness of the obtained channel impulse response estimate and the hard decisions of the data symbols. The BER curves show that those assumptions are valid for moderate to high $E_{s} / N_{0}$ values.

For the coded case, we have considered a turbo code that consists of two identical 16-state rate $1 / 2$ recursive systematic convolutional codes with generator sequences $(37)_{8}$ and $(21)_{8}$ (in octal notation) through a uniform random interleaver of length 919 . The parity bits are punctured to obtain an overall rate of approximately $1 / 2$. Every iteration, the turbo decoder provides the necessary hard decisions on the transmitted data symbols for the decision-directed FO and channel estimation algorithm. After 9 iterations convergence is reached. The application of our proposed algorithm results in a small loss in performance compared to the BER of a receiver with perfect knowledge about the channel and the FO. The gap between the two curves is less than $1 \mathrm{~dB}$.

\section{CONClusion}

In this contribution, we have presented an iterative decisiondirected joint FO and channel estimation algorithm for KSPOFDM, which is initialized by pilot-aided FO estimation without channel knowledge.

Two initialization algorithms are considered: TD initialization and FD initialization. Both exploit the presence of the pilot carriers and the pilot symbols in the guard interval to estimate the FO. The initial FO estimate is used in the pilotbased channel estimation algorithm from [13] for obtaining an initial channel estimate. The MSE of both initialization algorithms shows an error floor for moderate to high SNR but FD initialization gives the better performance.

After the initialization phase, the decision-directed algorithm is applied which iterates between FO estimation, channel estimation and data detection. The channel estimation step is an extension of the algorithm from [13]: all the carriers are considered for channel estimation. The simulation results show a substantial gain for the MSE: the error floor which is present after applying one of the initialization algorithms is (almost) completely removed. The performance of the decision-directed algorithm is independent of which initialization method is used first. In terms of the BER, the performance of a receiver with perfect knowledge of the FO and the channel is reached after only one iteration in the uncoded case. For a coded system, there is a gap of less than $1 \mathrm{~dB}$ between the performance of a receiver with perfect knowledge of the FO and the channel and a receiver which applies our proposed algorithm.

\section{APPENDIX}

In this appendix we derive the MSE of the FO estimator from (15) in the case that only pilot symbols are transmitted. The estimate is given by

$$
\hat{\epsilon}_{p}=\frac{1}{2 \pi} \frac{N}{N+\nu} \frac{\angle(R(P))}{P},
$$

where $R(P)$ is defined in (12). First we rewrite $R(P)$ as

$$
\begin{aligned}
R(P)= & (K-P) e^{j 2 \pi \frac{P(N+\nu)}{N} \epsilon_{P}} \boldsymbol{\Gamma}^{H} \boldsymbol{\Gamma}(1 \\
+ & \frac{1}{(K-P) \boldsymbol{\Gamma}^{H} \boldsymbol{\Gamma}} \sum_{i=P}^{K-1} \boldsymbol{\Gamma}^{H} \mathbf{V}_{i} e^{-j 2 \pi \frac{i(N+\nu)}{N} \epsilon_{P}} \\
& \left.+\frac{1}{(K-P) \boldsymbol{\Gamma}^{H} \boldsymbol{\Gamma}} \sum_{i=0}^{K-P-1} \boldsymbol{\Gamma}^{T} \mathbf{V}_{i}^{*} e^{j 2 \pi \frac{i(N+\nu)}{N} \epsilon_{P}}\right)
\end{aligned}
$$

where we have neglected the second order noise terms.

For a complex number $x$ with $|x| \ll 1$, the function $\angle(1+x)$ is approximated by $\Im\{x\}$. We can use this approximation in (15) for sufficiently high $E_{s} / N_{0}$, which yields for $\hat{\epsilon}_{p}$

$$
\begin{aligned}
\hat{\epsilon}_{p}=\epsilon_{p}+\frac{1}{(K-P) P} \frac{1}{2 \pi} & \frac{N}{N+\nu} \frac{1}{\boldsymbol{\Gamma}^{H} \boldsymbol{\Gamma}} . \\
\Im\left\{\sum_{i=P}^{K-1} \boldsymbol{\Gamma}^{H} \mathbf{V}_{i} e^{-j 2 \pi \frac{i(N+\nu)}{N} \epsilon_{P}}\right. & \\
& \left.\quad+\sum_{i=0}^{K-P-1} \boldsymbol{\Gamma}^{T} \mathbf{V}_{i}^{*} e^{j 2 \pi \frac{i(N+\nu)}{N} \epsilon_{P}}\right\} .
\end{aligned}
$$

The MSE is given by

$$
\begin{aligned}
& E\left[\left|\hat{\epsilon}_{p}-\epsilon_{p}\right|^{2}\right]= \\
& \quad \frac{1}{(K-P)^{2} P} \frac{1}{(2 \pi)^{2}}\left(\frac{N}{N+\nu}\right)^{2} \frac{1}{\left(\boldsymbol{\Gamma}^{H} \boldsymbol{\Gamma}\right)^{2}} \boldsymbol{\Gamma}^{H} \mathbf{R}_{\mathbf{V}} \boldsymbol{\Gamma},
\end{aligned}
$$

for $P \leq K / 2$ and by

$$
\begin{aligned}
& E\left[\left|\hat{\epsilon}_{p}-\epsilon_{p}\right|^{2}\right]= \\
& \quad \frac{1}{(K-P) P^{2}} \frac{1}{(2 \pi)^{2}}\left(\frac{N}{N+\nu}\right)^{2} \frac{1}{\left(\boldsymbol{\Gamma}^{H} \boldsymbol{\Gamma}\right)^{2}} \boldsymbol{\Gamma}^{H} \mathbf{R}_{\mathbf{V}} \boldsymbol{\Gamma},
\end{aligned}
$$

for $P \geq K / 2$. The matrix $\mathbf{R}_{\mathbf{V}}$ is the autocorrelation matrix of the samples of the noise vector $\mathbf{V}_{i}: \mathbf{R}_{\mathbf{V}}=E\left[\mathbf{V}_{i} \mathbf{V}_{i}^{H}\right]$.

The MSE is symmetric with respect to $P=K / 2$ : the values $P=Q$ and $P=K-Q$ result in the same MSE, and the minimum value of the MSE is reached for the values of $P$ which are closest to $K / 3$ and $2 K / 3$. 


\section{ACKNOWLEDGMENT}

The authors wish to acknowledge the activity of the Network of Excellence in Wireless COMmunications NEW$\mathrm{COM}++$ of the European Commission (contract n. 216715) that motivated this work.

\section{REFERENCES}

[1] J. A. C. Bingham, "Multicarrier modulation for data transmission, an idea whose time has come," IEEE Commun. Mag., vol. 28, no. 5, pp. 5-14, May 1990.

[2] E. T. S. Institute, "Transmission and multiplexing (TM); access transmission systems on metallic access cables; very high speed digital subscriber line (VDSL); Part 2: Transceiver specification,” ETSI TS 101 270-2, Feb. 2001

[3] — "Digital audio broadcasting (DAB); DAB to mobile portable and fixed receivers," France, ETS 300 401, Feb. 1995.

[4] _ - "Digital video broadcasting (DVB); Framing structure, channel coding and modulation for 11/12 GHz satellite services," France, ETS 300 421, Dec. 1994.

[5] R. van Nee, G. Awater, M. Morikura, H. Takanashi, M. Webster, and K. W. Halford, "New high-rate wireless LAN standards," IEEE Commun. Mag., vol. 37, no. 12, pp. 82-88, Dec. 1999.

[6] B. Muquet, Z. Wang, G. B. Giannakis, M. de Courville, and P. Duhamel, "Cyclic prefixing or zero padding for wireless multicarrier transmissions," IEEE Trans. Commun., vol. 50, no. 12, pp. 2136-2148, Dec. 2002.

[7] H. Steendam and M. Moeneclaey, "Different guard interval techniques for OFDM: performance comparison," in Proc. 2007 Int. Workshop Multi-Carrier Spread Spectrum, pp. 11-24.

[8] L. Deneire, B. Gyselinckx, and M. Engels, "Training sequence versus cyclic prefix-a new look on single carrier communication," IEEE Commun. Lett., vol. 5, no. 7, pp. 292-294, July 2001.

[9] J.-J. van de Beek, M. Sandell, and P. O. Börjesson, "ML estimation of time and frequency offset in OFDM systems," IEEE Trans. Signal Process., vol. 45, no. 7, pp. pp. 1800-1805, July 1997.

[10] D. Van Welden, H. Steendam, and M. Moeneclaey, "Time delay estimation for KSP-OFDM systems in multipath fading channels," in Proc. 2009 Personal, Indoor Mobile Radio Commun. Symp.

[11] D. Van Welden and H. Steendam, "Near optimal iterative channel estimation for KSP-OFDM," IEEE Trans. Signal Process., vol. 58, no. 9, pp. 4948-4954, Sep. 2010.

[12] J. Song, Z. Yang, L. Yang, K. Gong, C. Pan, J. Wang, and Y. Wu, "Technical review on Chinese digital terrestrial television broadcasting standard and measurements on some working modes," IEEE Trans. Broadcast., vol. 53, no. 1, pp. 1-7, Mar. 2007.

[13] D. Van Welden, H. Steendam, and M. Moeneclaey, "Frequency-domain data-aided channel estimation for KSP-OFDM," in Proc. 2008 Int. Symp. Spread Spectrum Techniques Applications.

[14] T. Pollet, M. Van Bladel, and M. Moeneclaey, "BER sensitivity of OFDM systems to carrier frequency offset and Wiener phase noise," IEEE Trans. Commun., vol. 43, no. 234, pp. 191-193, Feb./Mar./Apr. 1995.

[15] L. He, F. Yang, C. Zhang, and Z. Wang, "Synchronization for TDSOFDM over multipath fading channels," IEEE Trans. Consumer Electron., vol. 56, no. 4, pp. 2141-2147, Nov. 2010.

[16] G. Liu and S. Zhidkov, "A composite PN-correlation based synchronizer for TDS-OFDM receiver," IEEE Trans. Broadcast., vol. 56, no. 1, pp. 77-85, Mar. 2010.

[17] J. Zheng and W. Zhu, "An algorithm for calibration of TDS-OFDM carrier frequency offset," IEEE Trans. Consumer Electron., vol. 55, no. 2, pp. 366-370, May 2009.
[18] F. Tufvessona and T. Maseng, "Pilot assisted channel estimation for OFDM in mobile cellular systems," in Proc. 1997 IEEE Veh. Tech. Conf., pp. 1639-1643.

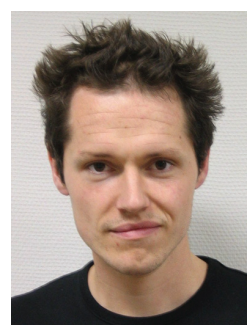

Dieter Van Welden (S'06) was born in Zottegem, Belgium, in 1982. He received the master's degree in electrical engineering and the Ph.D. in engineering, both from Ghent University, Belgium, in 2005 and 2012 , respectively. From 2005, he has been a researcher at the Department of Telecommunications and Information Processing, Ghent University. His main research interests are in statistical communication theory, carrier and symbol synchronization, coding and modulation, estimation and detection.

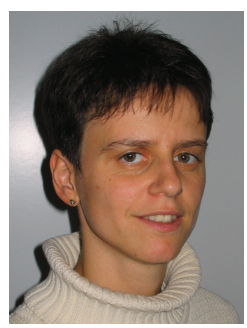

Heidi Steendam (M'01-SM'06) received the M.Sc. degree in electrical engineering and the Ph.D. degree in applied sciences from Ghent University, Gent, Belgium, in 1995 and 2000, respectively. Since September 1995, she has been with the Digital Communications (DIGCOM) Research Group, Department of Telecommunications and Information Processing (TELIN), Faculty of Engineering, Ghent University, Belgium, first in the framework of various research projects, and since October 2002, as a full time Professor in the area of digital communi-

cations.

Her main research interests are in statistical communication theory, carrier and symbol synchronization, bandwidth-efficient modulation and coding, spread-spectrum (multi-carrier spread-spectrum), satellite and mobile communication, cognitive radio and cooperative networks. She is the author of more than 100 scientific papers in international journals and conference proceedings.

Since 2002, she has been an Executive Committee Member of the IEEE Communications and Vehicular Technology Society Joint Chapter, Benelux Section, and since 2012, the vice chair. She has been active in various international conferences as Technical Program Committee Chair/Member and Session Chair. In 2004 and 2011, she was the conference chair of the IEEE Symposium on Communications and Vehicular Technology in the Benelux.

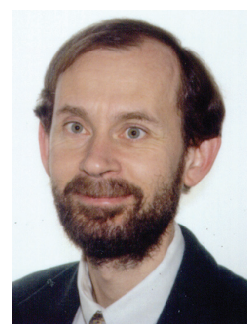

Marc Moeneclaey (M'93-SM'99-F'02) received the diploma of electrical engineering and the Ph.D. degree in electrical engineering from Ghent University, Gent, Belgium, in 1978 and 1983, respectively.

He is a Professor at the Department of Telecommunications and Information Processing (TELIN), Gent University. His main research interests are in statistical communication theory, (iterative) estimation and detection, carrier and symbol synchronization, bandwidth-efficient modulation and coding, spread-spectrum, satellite and mobile communication. He is the author of more than 400 scientific papers in international journals and conference proceedings. Together with Prof. H. Meyr (RWTH Aachen) and Dr. S. Fechtel (Siemens AG), he co-authored the book Digital Communication Receivers Synchronization, Channel Estimation, and Signal Processing (J. Wiley, 1998). $\mathrm{He}$ is co-recipient of the Mannesmann Innovations Prize 2000.

During the period 1992-1994, he was Editor for synchronization for the IEEE TRANSACTIONS ON COMMUNICATIONS. He served as co-guest editor for special issues of the Wireless Personal Communications Journal (on equalization and synchronization in wireless communications) and the IEEE JOURNAL ON SELECTED AREAS IN COMMUNICATIONS (on signal synchronization in digital transmission systems) in 1998 and 2001, respectively. 